\title{
The Significance of Context for the Emergence and Implementation of Research Evidence of Collaborative Problem-solving
}

\begin{abstract}
One of the fundamental purposes of educational research is to provide evidence to facilitate effective practice. However, the evidence itself does not have much value for practitioners unless key information about the context from which the evidence was generated is also provided. In this paper, we use the word 'context' to refer to factors that are relevant for learning including the interactions that learners experience with multiple people, artefacts, and environments. Unfortunately, in many educational research studies, either these factors do not get the required attention or information about them is presented in an incoherent structure. The resultant lack of information leads to two significant drawbacks. First, it creates confusion among practitioners who want to apply research evidence in their practice. Second, it leads to research studies that on the face of it are similar, but that in reality have resulted from evidence that has been collected in significantly different contexts being included under the same categories in reviews, meta-reviews and best evidence-syntheses. In this paper, we draw on the concept of 'relatability' of evidence and present taxonomy for collaborative problem-solving (CPS) that can be used to provide the valuable information against which research evidence can be indexed. By addressing the need for more detailed information about the contextual factors from which the evidence is generated to bridge the gap between research and practice in CPS research, we aim to exemplify the approach that is needed in educational research more generally.
\end{abstract}

\section{Introduction and Aims}

It is hard to argue against the potential value of evidence to inform and improve practice (Petty, 2009). Discussions around evidence in education emphasise that teachers should be focusing on 'what works', suggesting that practice should implement teaching methods that are presented to be effective at maximizing learning outcomes by research (Hattie, 2008). More specifically in Educational research in the UK, Department for Education recently allocated $£ 135$ million to improve evidenceinformed practice in education, highlighting the significance of the use and application of research evidence in schools (DfE, 2014). Within this context, there is a recent tendency in educational research to move towards the experimental research designs that aim to generate evidence to inform teaching practice while undervaluing the value of other type of research approaches. This movement towards positivist research methodologies in Education has led to heated debate over the last few years about the way in which evidence in Education is perceived, and the relationship between research and practice in educational research (de Bruin, 2015), with much discussion focusing on how to generate a positive relationship between educational research and teaching knowledge and practice (Pampaka, Williams, \& Homer, 2016).

In this paper, we focus on the gap between research and practice in Collaborative Problem-solving (CPS) (Luckin, Baines, Cukurova, \& Holmes, 2017) and argue that 
the 'what works' type of discussions in Education are not sufficient unless the contextual factors in which 'what works' question answered is also described. Here, we investigate research evidence on the effectiveness of CPS at reviews, metareviews and best evidence-syntheses level and present that the information within these reviews could not be differentiated according to the contextual factors that were in force when the research evidence was collected. By addressing the need for more detailed information about the context from which the evidence is generated in CPS research, we aim to stimulate the rethinking about evidence that is needed in educational research more generally.

This paper therefore has three main aims. The first aim is to highlight the importance of contextual factors in CPS domain to bridge the gap between research and practice. The second aim is to present what is considered as the existing evidence about CPS and the limitations of this evidence for educational practice. The third aim is to present a taxonomy of CPS to help understand variation across different contextual factors and increase evidence-informed implementations of CPS in practice. We start by highlighting the significance of contextual factors in CPS to address the gap between research and practice.

\section{The Significance of Contextual Factors to Bridge the Gap: the 'Relatability' of Evidence}

The omission of contextual factors is hard to understand when one considers that a wide range of research has illustrated that it is impossible to understand how people work or learn without also taking into account the people and artefacts that make up their context (Nardi, 1996 p. 38). Although, the term context is probably the term that is used most frequently within educational research papers to index the circumstances in which learning takes place (Cole, 1996), it is still a complex concept with its various definitions and interpretations by Educational researchers. It is very difficult to 'pin-down' in a way that enables context to be used as the basis for informing the practice of teaching and learning. However, context has been subjected to two principal conceptions: the first conceptualization is as 'that which surrounds', which is open to the criticism that context is portrayed as a container rather than part of the same situation. The second conceptualization requires that we interpret mind in a relational way: 'as distributed in the artefacts which are woven together and which weave together individual human actions in concert with and as part of the permeable, changing, events of life' (Cole et al., 1996). In this paper, our interpretation and use of the word context is similar to the latter conceptualization of context. Similarly, Manovich (2006) conceptualizes context for learners as being exposed to "a single context that is their lived experience of the world; a "phenomenological gestalt"'. In this sense, context is a reflection of the interactions that the learners have experienced with multiple people, artefacts, and environments. This interpretation of context includes a range of factors such as the learning tasks and the learning interactions of social constructivist learning processes, as well as its relatively simplistic interpretation as being that which surrounds learning and learners. It is sadly true that the treatment of context in educational studies, particularly when it is considered in this broader sense described above, is "under-examined, under-theorized and underdeveloped" (Gulson \& Symes, 2007). How could this situation have arisen when we 
know that context critically matters to learning; it defines a person's subjective and objective experience of the world in a spatially and historically contingent manner (Luckin, 2010). Why is context so poorly reported within the education research literature and how can we rectify this omission in a sustainable and systematic manner?

We argue that the omission of contextual information from much educational research devalues the impact of this research on the practice of educators. It also prevents the application of research findings to their own contexts, therefore prevents the "relatability" of them. Omission of contextual information prioritises description of educational interventions over their potential applied use. In this research paper, we present a conceptual framework and a taxonomy that can be used to provide appropriate contextual information about CPS interventions in a coherent and systematic way. The conceptual framework and the taxonomy presented here reflect the broader consideration of context discussed above.

The idea that the merit of research evidence for practitioners lies in its contextual information, is not novel in educational research. However, it is often overseen. Bassey (1981), in his comprehensive review of research into single events, introduced the concept of the 'relatability' of research and argued that the 'relatability' of research can be more meritorious than its generalizability. He contended if judged by the criterion of the usefulness to practitioners, the value of educational research is in the extent to which a practitioner reading it can relate it to her/his own teaching (or own situation to see whether it is applicable). The concept of 'relatability' is very useful when it comes to trying to make sense of the evidence in educational research. It is, however, a challenging concept. It requires the appropriate landscaping of complex related educational concepts, the clarification and definition of these concepts, and the classification of different types and examples of interventions. It requires some sense of systematization of complex educational interventions. All of which could only be achieved with a certain level of agreement among the experts in the field. However, it is clear to us that there is great value for all stakeholders in understanding the breadth and complexity of the CPS field, and the range and variety of CPS practices.

\section{Where does evidence regarding CPS come from in Educational research?}

As we mentioned in the introduction, researchers and practitioners in education often argue that they engage in and/or support evidence-informed practice. However, their views about what constitutes "evidence" vary broadly. This variance brings more confusion into the already complex endeavor of educational practice, and decreases the likelihood of employing evidence-informed practices in educational environments. To help with this situation educational researchers, psychologists, and learning scientists have presented different suggested solutions, including guidelines for rating the quality of individual studies, aggregating findings across studies, and classifying the overall level of empirical support for an intervention (Smith, 2013). Specifically in educational research, there are publications that provide explicit indicators of highquality studies, set a threshold for the number of such studies needed to establish that an intervention is evidence-informed, and delineate categories for these interventions that grade their efficacy (Kratochwill \& Stoiber, 2002; Odom et al., 2005). Most of 
the "evidence" in educational practice therefore appears to be coming from the results of meta-analyses and best-evidence syntheses.

Specifically for CPS, the vast majority of such meta-analyses and reviews of research to date tend to be based on studies examining cooperative and/or collaborative learning. There are clear distinctions drawn between such group work pedagogies in theory (See for instance Damon \& Phelps, 1989; Dillenbourg, 1999). However, these distinctions are rarely found in papers and reviews of empirical work. There have been no meta reviews or analyses of CPS exclusively, as far as we are aware. The large majority of meta-analyses bring together studies under the cooperative learning umbrella across multiple phases of education, with learners aged between 9 and 16 years in schools and older in universities. Nevertheless, meta-analyses tend to be melting pots of research on 'similar' topics undertaken in very different circumstances and conditions with the assumption that studies and effect sizes can be broken down into smaller chunks and then these effect sizes generalised. For instance, although quite problematic in practice, it would have been quite appropriate to combine effect sizes from 2 studies on collaborative learning relative to whole class teaching (maybe one was more on cooperative interaction) when one study was done in groups of 6 in high school on certain task types and the other was done in groups of 3 in primary school on other types of task. The only common element here is that people (adolescents and children were working together as opposed to listening to a teacher) but on all other dimensions there would be differences.

Once the generalisation has been made the assumption is that the result will happen regardless of the means of getting there, both in terms of the context and the process. This results in issues that even though well-established evidence regarding the impact of CPS on various learning outcomes exists, it is hard to argue that the evidence would be strong under every contextual factors or even so, its strength would be the same. Next, we review some of the most commonly cited reviews in the field and their limitations.

\section{Findings from the Meta-analytic evidence about collaborative and cooperative learning including CPS and their limitations}

Meta analyses are informative, they tell us a lot about the effectiveness of cooperative and collaborative approaches over other approaches to teaching and learning. However, the mixing of and comparison between often markedly different types of studies, across a wide range of educational circumstances that vary in many ways, for example, in education phase, curriculum area, culture, task demands, and in their different approaches to group work. The result is that meta-analyses in education, and in this instance in the general area of collaborative learning are not as informative as they appear on first look, particularly for practitioners.

Earlier systematic reviews and the best evidence synthesis from Slavin (1989) reported positive effects for cooperative learning methods on a range of outcome variables, such as achievement, socialization, motivation, attitudes towards subjects and studying, and personal self-development. Then, two significant reviews published in early 2000s (Johnson \& Johnson, 2002; Johnson, Johnson, \& Stanne, 2000) that provided marked effects $(0.58$ to 0.70$)$ in favour of cooperative learning in 
comparison to competitive and individual learning. More specifically in a metaanalysis of 117 studies that involved the Learning Together approach from the Johnsons, the authors (Johnson \& Johnson, 2002) found significant positive impact of collaborative learning on a range of academic and social outcomes. Furthermore, Johnson et al. (2000) investigated 164 research studies involving eight different cooperative learning strategies across different education levels (ages 7 through to adult) and found that the biggest effect size was achieved by the Learning Together approach for which the positive interdependence was the fundamental feature. This finding is particularly interesting for this paper as positive interdependence is also considered as a key requirement for CPS. Positive interdependence "exists when individuals perceive that they can reach their goals if and only if the other individuals with whom they are positively linked also reach their goals" (Johnson, Johnson, Roseth, \& Seob Shin, 2014, p.2). A range of factors may help to explain the different levels of effect sizes that is reported in these review studies. For example, contradictions and inconsistencies are often evident between different analyses and the bringing together of different measures on different scales and of different qualities does nothing to help matters. In addition to which, the calculation and comparison of effect sizes across studies is not an exact science in which a systematic study of the structure and behaviour of the certain interventions are observed. Therefore, they would retain the type two errors that involve incorrect retaining of false hypotheses in them, which leads to incorrect conclusions.

More recently, Roseth, Johnson, and Johnson (2008) tested a social-contextual view of the processes and mechanisms by which the benefits of cooperative learning are promoted. They reviewed 148 studies that compared the effectiveness of cooperative learning approaches to more traditional approaches in terms of its effect on promoting early adolescents' achievement and peer relationships. These studies represented over 8 decades of research on over 17,000 early adolescents from 11 countries and 4 multinational samples. The authors found that both higher achievement and more positive peer relationships were associated with learning approaches that involve social interdependence rather than more traditional competitive or individualistic approaches. To be included in the meta-analysis, research studies were evaluated against the following criteria: (a) Participants were middle school-age children, (b) the study specifically dealt with the effect of social interdependence on the dependent variable, (c) the study contained quantitative measures of the dependent variable, including sufficient information to calculate an ES, (d) the study reported sufficient information to make conclusions about the relative effectiveness of social interdependencies compared with a control group, (e) the targeted subject matter was academic, and (f) studies were written or summarized in English. Although, this is another example of a very carefully designed research study, due to the nature of implementing meta-reviews and best evidence syntheses, it considers some contextual factors, which would certainly affect the results under the same umbrella. For instance, it considers studies that were focusing on different ages of student, even though different age groups students may place different amounts of emphasis on peer relationships (Erikson, 1963, 1968).

A more recent meta-analysis examined the effects of 629 learning studies that involved positive interdependence, as in CPS practice, across primary, secondary and tertiary education, representing 26 different countries, in comparison to traditional approaches in which there was either no interdependence or negative interdependence 
in relation to motivation and achievement. The authors (Johnson et al., 2014) found that learning approaches involving positive interdependence led to higher motivation (broadly defined including value, expectancy of success, engagement, intrinsic motivation etc.) and achievement than other approaches to instruction. Similarly, a meta-analysis by Kyndt et al. (2013) of 65 studies since 1995, again across primary, secondary and tertiary levels of education replicated the moderate to large effect sizes for achievement and attitudes that earlier and older meta-analyses had found. Both of these example reviews for instance consider, different academic subjects, which have different learning outcomes, learning tasks and perceived difficulties for students under the same evaluation. All of these factors would vary students' problem-solving abilities. Therefore, the value of these studies for practitioners, who would like to take evidence-informed decisions within their CPS teaching contexts, is little. By relying on such evidence as the only source for evidence-informed practice, we are at the risk of offering practitioners isolated facts as 'tips' for their practice without providing the important information of 'under what contextual factors exactly' the tip works and 'why'.

Moreover, more specific to national contexts, some reviews are less relevant to a specific nation's educational context than others. For example, a substantive review by Lou et al. (1996) combines research on group instruction, cooperative learning and within class grouping under the broad heading of 'within class grouping' which is compared to the effects of learning in classes that were not organised into groups. Such a general comparison is not particularly helpful in relation to UK schools since the vast majority of classrooms organise children and young people into groups, pairs or rows (see for instance Baines, Blatchford, \& Kutnick, 2003) rather than the individual seating and whole class teaching approaches that are prevalent in the US.

There are also a number of limitations associated with the studies included in metaanalyses in general. Probably the most fundamental limitation relates to the authenticity of the studies. The studies of collaborative and cooperative learning that are often included in reviews and meta-analyses have often focused on short term pieces of work or particular task types, that are designed for the purpose, may be novel and appealing, and that are less relevant to the requirements of the particular curriculum. Teachers are required to implement only collaborative or cooperative learning approaches and not to undertake individual or whole class teaching. These collaborative or cooperative learning situations are not therefore entirely natural or authentic and do not address the full needs of learners, which might call for different forms of task or interaction in order to develop different types of knowledge. For example, whole class approaches may be more useful for the introduction of new understanding and individual/independent work might be better suited to practice tasks (or individual thinking in preparation for group tasks) before or even after group work takes place (Kutnick, 1994; Kutnick \& Blatchford, 2013). Collaborative learning may be more effective for particular types of learning tasks (such as the application of understanding and skills already partially developed). Acknowledging this then means that collaborative learning should be used in a strategic manner for particular types of tasks and for particular types of learning rather than for all elements of learning.

Importantly, the collaborative learning approaches impose collaborative group working and task structures that may not always meet the needs of teachers operating 
in every day classroom settings. Teachers may avoid collaborative learning because of the management problems that are likely to arise when trying to work with multiple groups (Doyle, 1986) and to consider the learning of all pupils.

As has already been discussed, the most reliable sources of evidence about CPS are embedded within the broader sphere of collaborative and cooperative learning. In addition to which the value of these sources of evidence is limited in its applicability to practice. We can also add to the problems associated with collating good quality evidence about CPS, the general criticisms of controlled studies already discussed, and the fact that educational contexts present far too many variables to be controlled, indeed some of these variables may in fact be impossible to control. Furthermore, it should not be forgotten that human subjects are autonomous and they play a large role in their unpredictable impact on many research results. Bearing these issues in mind, even experiments conducted successfully seem to provide results which are only valid for a specific group of people under specific conditions and it is difficult to draw conclusions that will apply in general. There are also issues that relate to the fact that most controlled studies test the average change within an intervention group and the analysis of change made by each subject is often ignored. Statistical metrics that merge data across studies (as in meta-reviews and best evidence syntheses) compound the problems in such controlled studies. Basically, these metrics represent an average of the average changes reported across different studies (Smith, 2013). To add even extra complications, the individual educational research studies included in the review or meta-analyses vary from one another in a range of ways. For example, the nature of the interventions and their protocols will be different, as will the profiles of the participant and the outcome measures. Most significantly, with respect to our previous discussions of contextual factors, as an important factor when it comes to evaluating the success of any educational intervention, there is very limited account taken of the different contexts of the individual research studies included in the review of metaanalyses.

In summary then, the overwhelming evidence from the reviews and meta-analyses of research evidence about collaborative and cooperative learning including CPS, highlights the huge potential of these approaches offer to education, at least for certain learning tasks. However, a more in-depth analysis of these analyses makes it clear that, the value of evidence generated from these studies is limited for practitioner and policy-makers. Therefore, we argue for a notion of "cautious transferability" of evidence rather than aiming for blanket generalizability. Our suggestion as an initial step towards such reorientation in the educational research to improve its impact on practice is to present research findings with their contextual factors. This would lead to 1) better "relatability" of research finding into practice and 2) more accurate categorisation of studies at meta level analyses. One possible way of presenting contextual factors of educational interventions in a coherent and systematic way is to generate a taxonomy that can index the interventions of the focal construct. We argue that the taxonomy we present would be beneficial for both researchers and practitioners in this domain. For researchers, it would support the presentation of sufficient information regarding the CPS interventions to enable a thorough and comparable distinction between studies. It would help other researchers to draw understanding of the way different factors combine as well as understanding how certain CPS interventions relate to certain learning outcomes. For practitioners, the taxonomy would be useful to enable transferability of existing evidence on CPS to 
their own practice. Next, we present our suggested taxonomy for CPS as well as how it could be implemented.

\section{A CPS Taxonomy}

The taxonomy we developed was informed by and developed on the basis of the literature on the CPS research and practice as well as our experience and expertise as teachers and educational researchers of the topic. The main aim of creating the taxonomy was to identify the contextual factors within which different research evidence could be situated and based on this situated evidence the practice of CPS could be improved.

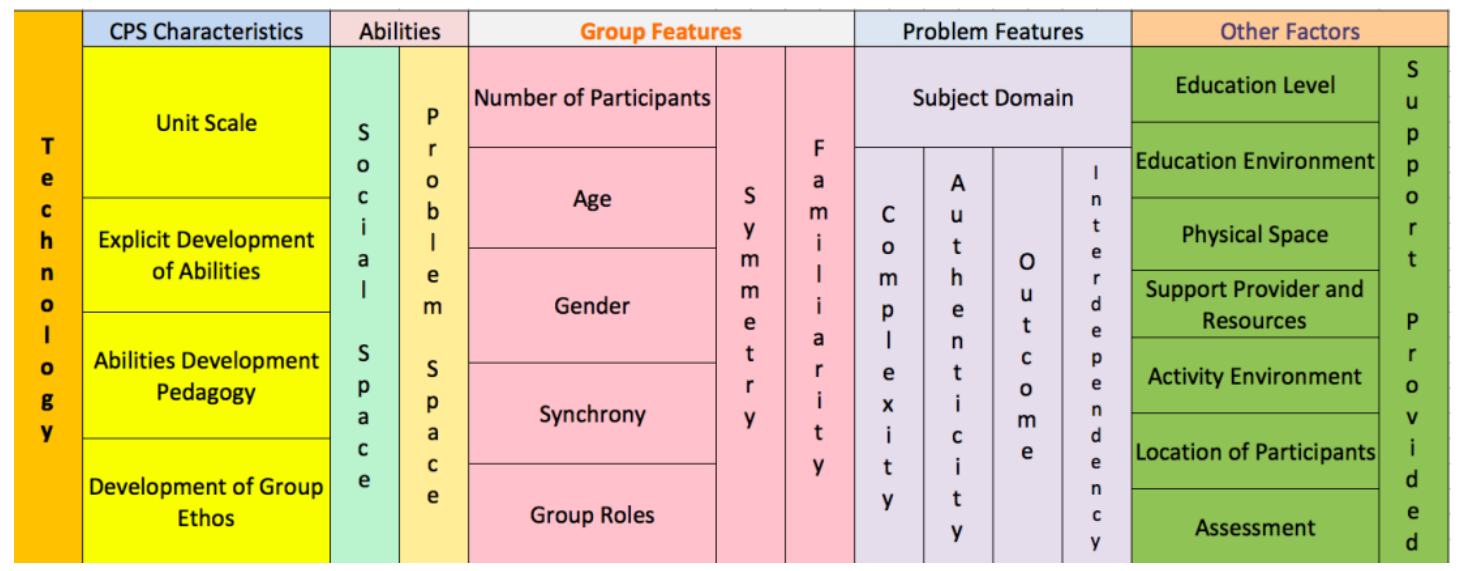

Fig. 1: A Potential Taxonomy for Collaborative Problem-solving

As can be seen in (fig. 1) the taxonomy we developed has six non-hierarchical, interconnected domains:

- Technology

- Characteristics of the CPS

- Abilities of the participants

- Group Features

- Problem Features

- The other factors

Each domain, except for the first, has multiple categories; each category has multiple taxonomic units. Each domain has multiple categories horizontal, vertical or both. Although it is very hard to make the separation, for the sake of simplifying the complex phenomenon of CPS while keeping the essential quarks of it in their place, we kept certain categories as nominal and others (which, we believe, are relatively harder to nominalize) as spectrum. So, horizontal categories represent nominal factors while vertical categories represent spectrum categories. For those spectra, we used high, medium, low taxonomic units and attempted to define them. These taxonomic units should be considered as suggestions rather than clear-cut categories.

\section{Technology}

Many types of technology can support CPS and the range is constantly evolving making for a dynamic array of possibilities. Technology can take the form of an interactive tabletop, a multi-player game or a discussion forum, for example. 
Technology can play the role of a tool to support collaboration, the means through which collaborators can communicate, a way of representing the knowledge and skills to be learnt or it can be embedded within the environment. It was deliberate decision not to sub-divide the technology domain in order to recognise the primary importance of the learning process and its participants and contextual factors. The technology should be subsidiary and enabling for effective pedagogies.

\section{Characteristics of the collaborative problem-solving design}

- Unit Scale: the size of the unit of CPS being considered.

O Single one off project/ activity

- Programme of interconnected CPS activities/ projects

- Development of participants' abilities: this captures the extent to which abilities are explicitly targeted by the CPS

- Not explicitly targeted

- Social space

- Problem space

- Social and Problem space

- How participants'/ whole group's abilities are developed: (any activity will involve the practice of a wide range of abilities simultaneously) this relates to the way that abilities are explicitly developed/taught separately from the CPS activity

○ Adult modelling

- Meta-cognitive/reflective processing (adult led)

- Meta-cognitive/reflective processing (group led)

○ Direct instruction (to include coaching)

- Development of group ethos:

○ Addressed through team building activities

- Addressed through Group processing

- Not addressed

\section{Abilities of the participants}

- Development of abilities related to social space: These abilities will be explicitly identified by the CPS and will aim to enable a person to function effectively while interacting with others (Ability to participate as a member of a group, Ability to place oneself in another's position, Ability to negotiate, Ability to resolve conflicts, misunderstandings, Ability to work effectively and respectfully with diverse teams, Ability to articulate ideas effectively (oral, written, non-verbal), Ability to listen effectively to decipher meaning.. etc.)

- Development of abilities related to problem space: These abilities will be explicitly identified by the CPS and will aim to enable a person to function effectively while working to bring a problem state closer to an aimed state. (Ability to identify facts, Ability to represent, formulate, and build knowledge, Ability to generate hypotheses, Ability to plan and execute 
actions, Ability to identify knowledge and skill deficiencies, Ability to reflect on actions. etc.)

Please note that the information relating to the process, or specific situational aspects of the practice is extremely hard to identify. However, the point here is to focus on the specific aims of the CPS and the structural design of the activity/programme as described by the creator or observed by the researcher.

\section{Group Features}

- Number of Participants: The number of participants forming a group.

- $\quad$ Age: Age distribution of participants forming a group.

○ Similar age groups

○ Mixed age groups

- Gender: Gender distribution of participants forming a group.

○ Same gender groups

$\bigcirc \quad$ Mixed gender groups

- Synchrony: Action timings of participants with respect to each other.

○ Participants are acting synchronously.

- Participants are acting asynchronously.

- Group roles: the extent to which participant roles are encouraged by activity design.

- Specialized roles allocated by activity designer

- Specialized roles encouraged (e.g. taking responsibility for subtask as in jigsaw working)

- Group roles unplanned (i.e. the group are free to plan for group roles)

- Group familiarity: Participants' experience of working with each other as part of the group.

o High: Participants work in experienced groups/ bonded groups.

- Medium: Acquaintance groups: participants are familiar and some may have worked together before.

○ Low: Participants may never have worked together before.

- Symmetry in the Group: Participants' proximity in terms of knowledge and skills related to the problem state.

- High: All participants have more or less the same level of knowledge and skills related to the problem state. (i.e. similar attainment group)

- Medium: Most of the participants have more or less the same level of knowledge and skills related to the problem state. (i.e. mixed attainment short distance)

- Low: Some/none of the participants have/has more or less the same level of knowledge and skills related to the problem 
state. (mixed attainment - substantial distance or distance unknown)

Please note that participants may have different but complementary pieces of information regarding the solution of the problem and this might be high or medium or low amount of information. This situation will contribute to the symmetry of the group.

\section{Problem Features}

- Subject Domain: Subject domain within the educational context.

$\bigcirc$ Science, Technology, Engineering and Maths

- Social Sciences

$\circ \quad$ Literacy, Arts and Humanities

- Cross curricular

- Intended Complexity: Proximity (judged by an expert in the domain and/or the teacher) of the problem state to the aimed state.

- High: Distance between the problem state and the aimed state is big

- Medium: Distance between the problem state and the aimed state is medium

- Low: Distance between the problem state and the aimed state is small

- Authenticity: Proximity of the problem to real-world problem examples.

o High: Participants are dealing with a real-world problem.

- Medium: Participants are dealing with a fabricated problem representing a real-world problem, a simplified version of a real-world problem, or a sub-problem of a real-world problem.

○ Low: Participants are dealing with a fabricated problem, which does not represent any real-world problem.

- Problem outcome: extent to which the problem is closed, best fit or open ended (judged by an expert in the domain and/or the teacher).

$\circ$ High: Single solution/closed problems, problem has a single solution

○ Medium: Multiple solutions/Best fit problems, problem has a number of possible solutions, which can be evaluated using success and quality criteria.

- High: Any solutions/ Open problems, there is no single solution or best fit - any solution or outcome (except maybe no outcome) is a solution

- Interdependency: extent to which the design characteristics leading to social interdependence.

○ High: Most/all of the design characteristics lead to social interdependence in the group.

○ Medium: Some of the design characteristics lead to social interdependence in the group. 
- Low: A few/none of the design characteristics lead to social interdependence in the group.

\section{The Other Factors}

- Education Level: Level of education of the participants

o Primary School

○ Secondary School

- Tertiary Education (FE and HE)

$\circ$ A group with mixed education levels

- Education Environment: Participants' physical space

○ Classroom

○ Fieldwork

○ School Laboratories

- Physical Space

- Flexible use of furniture/space for participant interaction explicitly considered and used

- Flexible use of furniture/space not considered or used

- Activity Environment: The interaction space

o Participants take actions to reach the aimed state in a digital environment (Building a tower in simulation environments).

- Participants take actions to reach the aimed state in the real world using physical models of the problem state (Building a small scale replica of a tower using models).

- Participants take actions to reach the aimed state in the real world problem state (Building the actual tower).

- Location of Participants: Location of the participants with respect to each other.

- Participants are in the same physical environment

○ Participants are in different physical environments.

- Assessment

- Participants are assessed on their performance individually.

- Participants are assessed on their performance as a group.

- Participants are monitored on their performance individually, but not assessed.

- Participants are monitored on their performance as a group, but not assessed.

- Participants are not monitored or assessed on their performance.

- Identity of support provider: identity of the support provided

- Trained expert

- Assistant/mentor (teaching assistant or learning support assistant)

- Digital tool/context 
○ Physical tool/context

- $\quad$ Resources and tools: tools or resources for assisting with the CPS

○ Only digital tools/resources used

- Only physical tools/resources used

- Both digital and physical resources used

- Support Provided: Amount and quality of support provided to participants with digital tools and/or adult support.

○ High: Scaffolded support for both the social and the problem space is provided.

- Medium: Scaffolded support is provided either in the social or the problem space.

- Low: Scaffolded support not provided for the social or the problem space.

\section{Application of the Taxonomy}

The taxonomy was designed to present a list of the potential contextual factors that impact on CPS that was broad enough to encompass a wide range of the activities that can be considered as CPS practice and yet, specific enough for practitioners to relate the evidence generated to their own teaching contexts. In order to exemplify the use of the taxonomy, we provide a recent example extracted from the literature below.

"In this activity, the teacher organizes a role-play as a summative performance task, where he hosted a counter-factual conference. Each student was assigned one of the five European nations that they were to represent at the conference. The teacher set their desks in the shape of a horse-shoe and the students were seated according to their country groups, with a name-tag of their country placed in front of each group. As a host of the conference, he placed himself in the centre and orchestrated the students' interactions from that position. Each group shared a tablet where they organised their individual microblogs, deleted those that were redundant and wrote new blogs. By toggling the "sharing" button on the tablet, their microblogs were viewed on the whiteboard in front of the class. In most groups, the students took turns and they all contributed to some degree."

This specific example was chosen as it is one of those research studies, which actually provides relatively more detailed information about the contextual factors of the CPS intervention being investigated. If we apply the taxonomy to the practice example the "CPS intervention identity chart" would look like table 1 below.

\begin{tabular}{|c|c|}
\hline Characteristics of CPS & 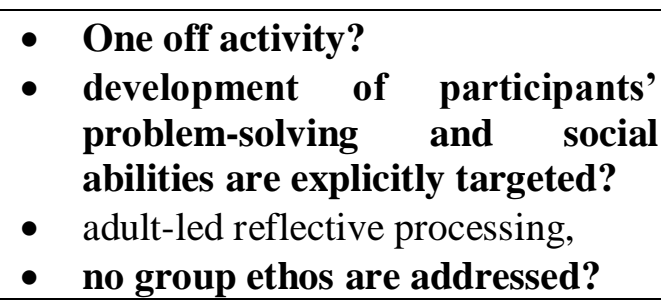 \\
\hline & $\begin{array}{l}\text { - Groups of five people of similar } \\
\text { age? } \\
\text { - mixed gender groups? }\end{array}$ \\
\hline
\end{tabular}




\begin{tabular}{|c|c|}
\hline Group Features & 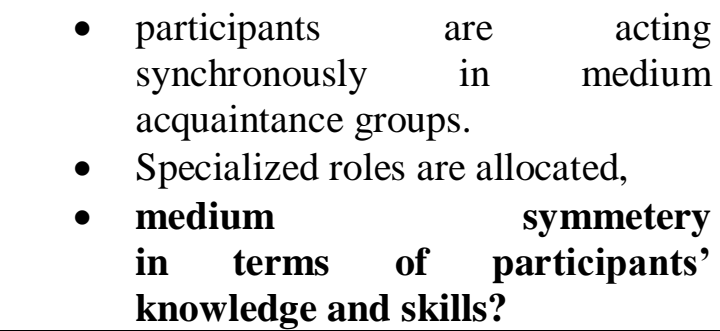 \\
\hline Problem features & $\begin{array}{l}\text { Social Science domain, } \\
\text { - high complexity, high } \\
\text { authenticity, and medium } \\
\text { outcome of the problem? } \\
\text { medium social interdependence } \\
\text { of participants? }\end{array}$ \\
\hline The Other Factors & $\begin{array}{l}\text { - Secondary level education, } \\
\text { - classroom settings, } \\
\text { - flexible use of furniture/space } \\
\text { explicitly considered, } \\
\text { - participants are in the same } \\
\text { physical space and taking actions in } \\
\text { a digital environment and in real } \\
\text { world, } \\
\text { - participants are evaluated } \\
\text { individualy? } \\
\text { provided high level of support by } \\
\text { the trained expert in both social } \\
\text { and problem spaces? }\end{array}$ \\
\hline Problem and Social Space & $\begin{array}{l}\text { Problem and social space explicitly } \\
\text { targeted? }\end{array}$ \\
\hline Technology & Internet, wiki, tablets, microblogs \\
\hline
\end{tabular}

Table 1: CPS activity identity card

We have already highlighted the fact that not all the contextual information about the CPS interventions researched are provided explicitly. In fact, most of the relevant and important information is not provided. The bold type in Table 1 is used to identify the information that is left to the interpretation of the reader. For instance, there is no information about the evaluation of the activity, or what abilities the activity aims to develop through the CPS activity. Information about the social interdependence of students is left to the interpretation of the readers and support provided by the teacher is not explicitly presented. Students' symmetry in their knowledge is not provided, neither are the authenticity, complexity, or the outcome of the problem is presented. Most of these factors would have been considered by the researchers and practitioners (or should have had), however they are implicit in the reports of the empirical work and are not made explicit in the research studies. This ambiguity in evaluation of studies then effect the potential implementation of the investigated research interventions in further practice as well as diminishing the quality of further metastudies that involve them. We argue that the taxonomy that we provide in this paper can be used to make these implicit factors explicit and to help clarify the evidence available from research about CPS and bridge the gap between research and practice. 


\section{Conclusions}

In most scientific communities, the systematic and statistical accumulation of results from experimental research studies is considered as the 'gold standard' of evidence for practice. We have argued in this paper that there are two key dangers in privileging only this kind of research evidence: 1) Its relative value to practitioners is limited unless the contextual factors from which the evidence generated is also provided. 2) It leads to research studies that on the face of it are similar, but that in reality have significant differences. This results evidence collected in significantly different contexts, being included under the same categories at meta-level investigations, which blurs the quality and value of the evidence.

We argue that more information about the contextual landscaping of the interventions should be provided for practitioners and policy-makers to enable the latter to make more informed policy decisions, and to enable the first to develop their evidenceinformed practices. For this purpose, we have presented a taxonomy of CPS, which we believe can be the initial step towards the creation of a common language that can be used and shared by both practitioners and researchers in this field. We think that the CPS taxonomy provided can be used to provide information relative to all of these areas and thus enabling understanding and "relatability" of research findings to their own contexts. It can also be used to create more accurate categories of studies at meta level investigations and reliable evidence. The taxonomy aims to remind practitioners and researchers some of the fundamental aspects while creating, evaluating and interpreting the practice of CPS. However, by addressing the need for more detailed information about the contextual factors in CPS research, we aim to raise awareness to the need that is required in educational research more generally.

\section{References}

Baines, E., Blatchford, P., \& Kutnick, P. (2003). Changes in grouping practice over primary and secondary school. International Journal of Educational Research, 39, 9-34.

Bassey, M. (1981). Pedagogic research: On the relative merits of search for generalisation and study of single events. Oxford Review of Education, 7(1), 73-94.

Cole, K. C., VanTilburg, D., Burch-Vernon, A., \& Riccio, D. C. (1996). The importance of context in the US preexposure effect in CTA: Novel versus latently inhibited contextual stimuli. Learning and Motivation, 27(4), 362374.

Damon, W., \& Phelps, E. (1989). Critical distinctions among three approaches to peer education. International Journal of Educational Research, 13(1), 9-19. doi:http://dx.doi.org/10.1016/0883-0355(89)90013-X

De Bruin, C. L. (2015). Conceptualizing effectiveness in disability research. International Journal of Research \& Method in Education. doi:10.1080/1743727X.2015.1033391

Department for Education. (2014). School funding: Research priorities and questions. Crown copyright.

Dillenbourg, P. (1999). What do you mean by 'collaborative learning'? Cognitive and Computational Approaches, 1-19.

Erikson, E. H. (1963). Childhood and society, New York: Norton. 
Erikson, E. H. (1968). Womanhood and the inner space. Identity, youth and crisis, 261-294.

Doyle, W. (1986). Classroom organization and management. In M. C. Wittrock (Ed.), Handbook of research on teaching. New York: Macmillan.

Gulson, K. N., \& Symes, C. (2007). Spatial theories of education: Policy and geography matters: Routledge.

Hattie, J. (2008). Visible learning: A synthesis of over 800 meta analyses related to achievement. New York, NY: Routledge.

Johnson, D. W., \& Johnson, R. T. (2002). Learning together and alone: Overview and metaanalysis. Asia Pacific Journal of Education, 22(95-105).

Johnson, D. W., Johnson, R. T., Roseth, C. J., \& Seob Shin, T. (2014). The relationship between motivation and achievement in interdependent situations. Journal of Applied Social Psychology, 44(9), 622-633.

Johnson, D. W., Johnson, R. T., \& Stanne, M. E. (2000). Cooperative learning methods: A meta-analysis. Minneapolis: University of Minnesota, Cooperative Learning Center.

Kratochwill, T. R., \& Stoiber, K. C. (2002). Evidence-based interventions in school psychology. School Psychology Quarterly, 17, 341-389.

Kutnick, P. (1994). Use and effectiveness of groups in classrooms. In P. Kutnick \& C. Rogers (Eds.), Groups in schools. London: Cassell.

Kutnick, P., \& Blatchford, P. (2013). Effective group-work in Primary school classrooms: the SPRinG approach. Dordrecht: Springer.

Kyndt, E., Raes, E., Lismont, B., Timmers, F., Cascallar, E., \& Dochy, F. (2013). A meta-analysis of the effects of face-to-face cooperative learning: Do recent students verify or falsify earlier findings? Educational Research Review, 10, 133-149.

Lou, Y., Abrami, J. C., Spence, C., Poulson, B., Chambers, S., \& d'Appolonia, S. (1996). Within Class grouping: a meta-analysis. Review of Educational Research, 66, 423-458.

Luckin, R. (2010). Re-designing Learning Contexts: Technology-Rich, LearnerCentred Ecologies. London: Routledge.

Luckin, R., Baines, E., Cukurova, M., Holmes, W. (2017). Solved! Making the case for collaborative problem-solving. NESTA: London, UK.

Nardi, B. A. (1996). Studying context: A comparison of activity theory, situated action models, and distributed cognition. Context and consciousness: Activity theory and human-computer interaction, 69-102.

Odom, S. L., Brantlinger, E., Gersten, R., Horner, R., Thompson, B., \& Harris, K. (2005). Research in special education: Scientific methods and evidencebased practices. Exceptional Children, 71, 137-148.

Pampaka, M., Williams, J., \& Homer, M. (2016). Is the educational 'what works' agenda working? Critical methodological developments. International Journal of Research and Method in Education, 39, 231-236.

Petty, G. (2009). Evidence-based teaching: A practical approach: Nelson Thornes.

Roseth, C. J., Johnson, D. W., \& Johnson, R. T. (2008). Promoting early adolescents' achievement and peer relationships: the effects of cooperative, competitive, and individualistic goal structures. Psychological Bulletin, 134(2), 223. 
Slavin, R. E. (1989). Cooperative learning and student achievement. In R. E. Slavin (Ed.), School and classroom organization (pp. 129-156). New Jersey: Lawrence Erlbaum.

Smith, T. (2013). What is evidence-based behaviour analysis? The Behavior Analyst, 36(1), 7-33. 